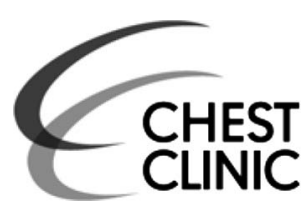

${ }^{1}$ Cardiothoracic Surgery Department, NHS National Waiting Times Centre Board, Golden Jubilee National Hospital, Clydebank, Glasgow, UK

${ }^{2}$ Radiology Department, Glasgow Royal Infirmary, Glasgow, UK

\section{Correspondence to} Karim Hani Kamel Morcos, Cardiothoracic Surgery Department, NHS National Waiting Times Centre Board, Golden Jubilee National Hospital, Agamemnon Street, Clydebank, Glasgow G81 4DY, UK; karimkamel7@gmail.com

Received 14 October 2012 Revised 15 November 2012 Accepted 31 December 2012 Published Online First 24 January 2013

\title{
Life-threatening haemoptysis due to tracheal varices secondary to pulmonary vein obstruction
}

\author{
Karim Hani Kamel Morcos, ${ }^{1}$ Giles Roditi, ${ }^{2}$ Stewart Craig ${ }^{1}$
}

\section{HISTORY}

A 30-year-old man with a 3 day history of increasing breathlessness and frank haemoptysis was suspected of having pulmonary embolism and anticoagulated with heparin. Haemoptysis worsened so anticoagulation was reversed and he was transferred to our institution.

Pulmonary and bronchial angiography revealed a small right pulmonary artery and a prominent right intercostobronchial trunk which was embolised. However, haemoptysis recurred and a contrast enhanced ECG-gated CT scan confirmed a hypoplastic right pulmonary artery and showed thrombosed right pulmonary veins that had no communication to the left atrium, a 'bulky' right hilum and thickened bronchial walls with smooth mucosal indentations into the airway lumen (figures 1 and 2). Bronchoscopy confirmed carinal and right bronchial varices. A ventilation perfusion $(\mathrm{V} / \mathrm{Q})$ scan showed normal ventilation but very little perfusion of the right lung.

The patient underwent a standard dissectional stapled right pneumonectomy. Intraoperatively, the right main bronchus and distal trachea were encircled by bronchial veins which were the only means by which the lung could drain as the native pulmonary veins had no discernable flow. In addition there were a number of venous collaterals to

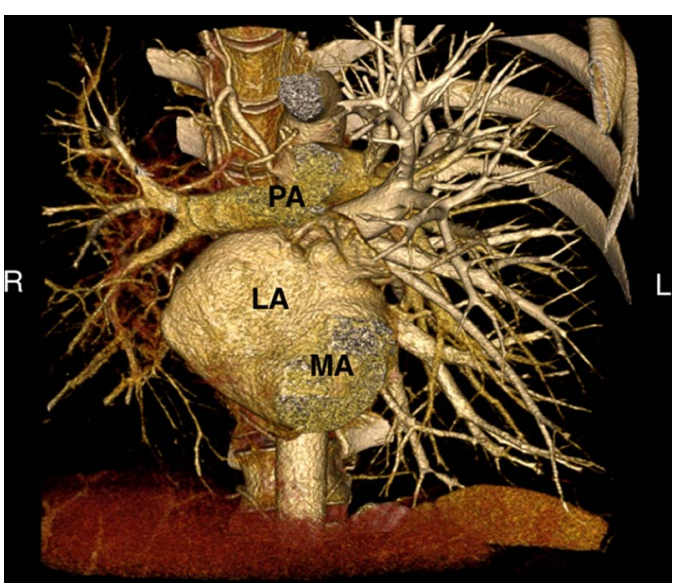

Figure 1 Volume rendered CT image of heart and great vessels viewed from anterior with right heart chambers, right ventricular outflow tract (RVOT) and pulmonary trunk back to pulmonary artery bifurcation plus left ventricle (LV) back to mitral annulus level removed for clarity in order to show left atrium and connections. The right pulmonary arterial tree is hypoplastic compared with the left side and there are no enhancing pulmonary veins on the right $L A$, left atrium; MA, mitral annulus; PA, pulmonary artery. Access the article online to view this figure in colour.

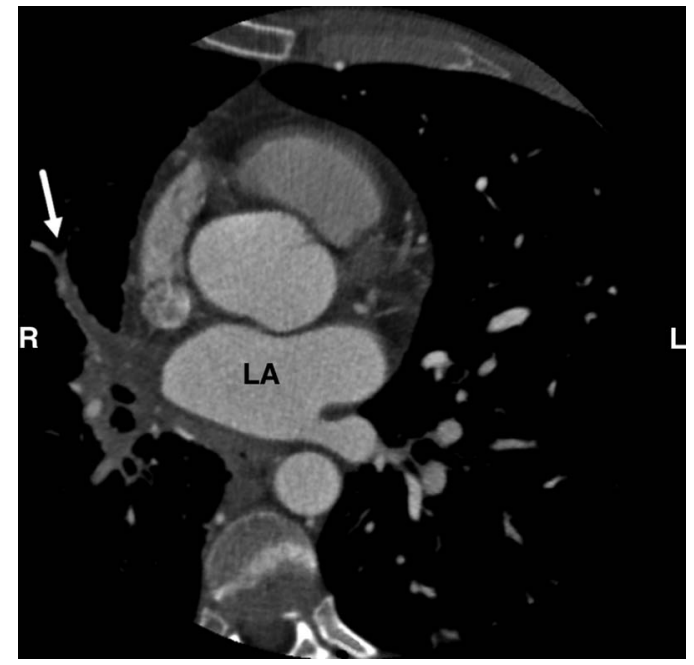

Figure 2 Transverse CT image showing left atrium with enhanced left pulmonary venous tributaries but none on the right side where the atretic thrombosed pulmonary veins are not enhanced (white arrow).

the chest wall. Postoperatively the patient had no further haemoptysis and repeat bronchoscopy showed resolution of the varices.

Histopathology showed thrombosed pulmonary veins with signs of recanalisation and a dilated bronchial venous plexus involving the hilar bronchial submucosa. There was an acquired moderate degree of chronic inflammation involving the pulmonary artery wall causing stenosis.

\section{DISCUSSION}

Tracheal varices from pulmonary venous occlusive disease may cause massive haemoptysis. ${ }^{1}$ Cases with similar findings to ours have been reported but with a different aetiology; idiopathic hilar fibrosis causing pulmonary vein occlusion. ${ }^{2}$

In our patient there was no hilar fibrosis and the aetiology of the pulmonary venous thrombosis remains unclear. The prominent collateral circulation within the walls of the airways were tracheal varices caused by pulmonary venous thrombosis.

\section{Learning point}

Massive haemoptysis can occur secondary to non-embolic pathologies and this should be considered in young patients with no risk factors for pulmonary embolism in whom early systemic anticoagulation may be life threatening. 
Contributors KHKM: patient's follow-up, literature search and writing of the manuscript. GR: CT scan images and writing of the manuscript. SC: surgeon who performed the operation, patient's follow-up and writing of the manuscript.

Competing interests None.

Patient consent Obtained.

Provenance and peer review Not commissioned; externally peer reviewed.

\section{REFERENCES}

1 Lozano LA Sosa, Shahir K, Akbar M, et al. A case of tracheal varices: an unusual but important cause of mural nodules in the trachea. Br J Radiol 2011;84: e62-4.

2 den Bakker MA, Thomeer M, Maat AP, et al. Life-threatening hemoptysis caused by chronic idiopathic pulmonary hilar fibrosis with unilateral pulmonary vein occlusion. Ann Diagn Pathol 2005;9:319-22. 